\title{
Regulation of Human MUC7 Mucin Gene Expression by Cigarette Smoke Extract or Cigarette Smoke and Pseudomonas aeruginosa Lipopolysaccharide in Human Airway Epithelial Cells and in MUC7 Transgenic Mice
}

\author{
Hao Fan and Libuse A. Bobek \\ Department of Oral Biology, University at Buffalo, The State University of New York, 109 Foster Hall, Buffalo, NY \\ 14214, USA
}

\begin{abstract}
Objective: The human MUC7 gene encodes a low-molecular-weight mucin glycoprotein that functions in lubrication/protection of epithelial surfaces of the oral cavity and respiratory tract. This study was designed to evaluate the effect of cigarette smoke extract (CSE), cigarette smoke (CS), and Pseudomonas aeruginosa lipopolysaccharide (LPS), either alone or in the combination, on MUC7 expression in vitro and in vivo.

Materials and Methods: qRT-PCR was used to determine the levels of mucin gene transcription in the human lung carcinoma cell line NCI-H292 (in vitro) and MUC7 transgenic mouse tissues (in vivo). ELISA was used to assess mucin glycoprotein levels in the cell line, and immunohistochemistry to assess mucins in lung and trachea sections.

Results: In vitro treatment of cells with LPS $(10 \mu \mathrm{g} / \mathrm{ml})$ or CSE $(0.5,1,2.5$ and $5 \%)$ alone, resulted in a statistically significant increase of MUC7 transcripts only with 1\%CSE (3.2-fold). The combined CSE/LPS treatment resulted in a synergistic increase of MUC7 with $0.5 \%$ CSE/LPS (4.4 fold). MUC7 glycoprotein levels increased only minimally, the highest increase was seen with the $0.5 \% \mathrm{CSE} / \mathrm{LPS}$ combination treatment (1.3-fold). In vivo exposure of MUC7 transgenic mice to CS, LPS or CS/LPS combination resulted in significant increase in MUC7 transcripts only with LPS treatment (in both trachea and lung). Immunohistochemistry indicated variable increase in MUC7 glycoprotein with CS and LPS treatment, both in the trachea and lungs, but CS/LPS exposure appeared to yield the highest increase.
\end{abstract}

Conclusion: In vitro, CSE and a combination of CSE/LPS treatment upregulated MUC7 gene transcription. In vivo, LPS upregulated MUC7 transcription, and a combination of CS/LPS appeared to increase MUC7 glycoprotein.

Keywords: MUC7 expression, cigarette smoke, LPS, NCI-H292 cells, lungs, trachea.

\section{BACKGROUND}

In the respiratory tract, mucus has an important protective function by entrapping inhaled foreign debris and bacteria, and clearing them from the airways by ciliary action [1]. Mucin production and secretion by specialized epithelial cells is a common mechanism used by mammals to protect the underlying mucosae against various injuries and pathogenic organisms. However, excess mucin production overwhelms the mucociliary clearance, resulting in defective mucosal defense and contributing to morbidity and mortality in related diseases. Acute challenges to the respiratory tract (including environment toxins, allergens, or infectious pathogens) activate lung inflammatory/immune response mediators. Some mediators initiate mucin hypersecretion by activating secretory granules of goblet cells in the surface epithelium and/or secretory cells in the submucosal gland [2]. A large number of biologically active molecules, such as cytokines, bacterial products, growth factors, differentiation agents and other factors, have been shown to regulate mucin

*Address correspondence to this author at the Department of Oral Biology, University at Buffalo, 109 Foster Hall, Buffalo, NY 14214, USA; Tel: 716829-2465; Fax: 716-829-3942; E-mail: lbobek@buffalo.edu synthesis (in vitro and/or in vivo) in various cell types. The notion that inflammation activated mucin gene expression has had wide-reaching ramifications has emerged some time ago. It focused on the importance of mucin gene regulation in airway diseases such as cystic fibrosis, chronic obstructive pulmonary disease (COPD) and asthma [3].

Smoking is among the major environmental health risk factors in the modern world affecting almost all the organ systems in the human body. Cigarette smoking is a major etiological factor in the pathogenesis of chronic obstructive pulmonary disease (COPD) [4]. Oxidative stress caused by cigarette smoking is thought to induce a chronic inflammatory response in the lungs, which results in the destruction of the alveolar cell walls and emphysema [5]. Moreover, increased oxidative stress can trigger proinflammatory cytokines, which are increased in the lungs of smokers and patients with COPD [6]. One of the previous studies have shown that cigarette smoke, along with other pathogenic factors, such tumor necrosis factor and LPS, can synergistically enhance respiratory MUC5AC induction [7].

The human MUC7 gene encodes a small mucin glycoprotein that is secreted mainly by salivary glands, but the gene is also expressed in tracheobronchial system [8-10]. 
MUC7 main functions are thought to be promoting the clearance of bacteria; MUC7 has been shown to interact with a variety of bacteria, including four strains of streptococci, Actinobacillus actinomycetemcomitans, Staphylococcus aureus and Pseudomonas aeruginosa [11, 12], and to aid in mastication, speech and swallowing. In terms of MUC7 gene regulation, previous studies in our laboratory have shown that MUC7 transcript and glycoprotein product were increased in vitro upon treatment of airway epithelial cells A549 with a panel of cytokines (IL-1 $\beta$, IL-4, IL-13, TNF- $\alpha$, EGF), and in vivo upon the treatment of MUC7 transgenic mice with LPS [13].

The study described in this manuscript was designed to further explore the regulation of MUC7 gene expression both in vitro and in vivo. Expression of human MUC5AC, mouse Muc5ac and mouse Muc10 (MUC7 ortholog) was assessed for comparative purposes. The results were compared and contrasted to the results of previous in vitro and in vivo studies. The in vitro experiments evaluated the effect of cigarette smoke extract (CSE), and CSE in combination with Pseudomonas aeruginosa lipopolysaccharide (LPS) on MUC7 expression in human lung mucoepidermoid carcinoma cell line NCI-H292. The in vivo experiments evaluated the effect of cigarette smoke (CS), and CS and LPS combination on MUC7 expression utilizing MUC7 transgenic mice.

\section{METHODS}

\section{Preparation of Cigarette Smoke Extract}

Aqueous cigarette smoke extract (CSE) was used to mimic the effects of cigarette smoke in the experiments with the cell line. Cigarette smoke of Research Cigarettes 1R1 (University of Kentucky) was slowly bubbled through the Phenol Red-free RPMI 1640 medium containing $10 \mathrm{mM}$ HEPES. The suspension ( $\mathrm{pH}$ adjusted to 7.4) was then filtered through a $0.22 \mu \mathrm{m}$ pore filter to remove bacteria and large particles. The resulting CSE from one cigarette in 30 $\mathrm{ml}$ of the media, considered to be $100 \%$, was then diluted to different concentrations (using the same medium), and then applied to cell cultures within 30 min of preparation (either alone or in combination with LPS).

\section{Cell Culture}

The human pulmonary mucoepidermoid carcinoma cell line NCI-H292 was obtained from the ATCC (American Type Culture Collection, Rockville, MD). The cells were grown in RPMI 1640 medium (GIBCO-BRL, Grand Island, $\mathrm{NY}$ ) with $2 \mathrm{mM}$ glutamine, $4.5 \mathrm{~g} / \mathrm{L}$ glucose, $10 \mathrm{mM}$ HEPES, $1 \mathrm{mM}$ sodium pyruvate; supplemented with $10 \%$ fetal bovine serum (GIBCO-BRL), penicillin (100 units $/ \mathrm{ml})$, and streptomycin $(50 \mathrm{mg} / \mathrm{ml})$, and maintained at $37^{\circ} \mathrm{C}$ in a $5 \%$ $\mathrm{CO}_{2}$ incubator. For different treatments, the cells were cultured in a $24-w e l l$ plate. At about $70 \%$ confluency, the cells were exposed to LPS $(10 \mu \mathrm{g} / \mathrm{ml}$; an optimal dose, based on the dose-response curve determined previously [13]), or CSE $(0.5 \%, 1 \%, 2.5 \%, 5 \%)$, or a combination of LPS and CSE. After 24 hours, the cells were harvested for RNA and cell lysate preparations, as described below.

\section{In Vivo Experiments with Mice}

Animal experiments were approved by the Institutional Animal Care and Use Committee at the State University of New York at Buffalo. MUC7 transgenic mice and nontransgenic mice littermates (produced in our animal facility), both males and females, were used in this experiment. All mice were 8 weeks old and had similar body weights $(\sim 25$ g). The mice that were exposed to cigarette smoke (CS) were exposed to it for 4 consecutive days, but only during the daylight hours ( 8 hours, 1 cigarette per hour). The selected time-course of the study was based on the previous study with the rat model of LPS-induced inflammation with concurrent cigarette smoke inhalation [7], except the number of cigarettes per day was reduced to 8 (from 24 in the rat model). A peristaltic pump was put inside a chamber under the fume hood with the mice. The CS was combusted through the pump at a low speed.

The experiment included the following 4 groups with 5 mice in each group. Group 1: Mice exposed to the CS only. Group 2: Mice exposed to CS/LPS. These mice were first exposed to CS for 24 hours, followed by instillation of LPS. Mice were anesthetized by injection of ketamine hydrochloride $(80 \mathrm{mg} / \mathrm{kg})$ and xylazine hydrochloride $(5 \mathrm{mg} / \mathrm{kg})$ to circumvent the cough reflex during instillation. The upper portion of trachea was surgically exposed (by making an incision in the skin and separating the thyroid gland overlying muscles and connecting tissues). A microsyringe carring a 25 -gauge soft needle filled with $10 \mu 1$ $(2.5 \mu \mathrm{g})$ of LPS solution was inserted into the exposed trachea and the solution was injected into the lumen of mouse trachea. After injection, and a few hours of recovery, the mice were exposed to CS for 3 more days. Group 3: Mice exposed to LPS. These mice were first exposed to air for twenty-four hours, followed by instillation of LPS, and 3 more days of exposure to air. Group 4: Mice exposed to air only. For all groups, on day 5, the mice were sacrificed and tissues (trachea and lungs) were collected for RNA isolation and immunohistochemistry analysis.

\section{RNA Preparation}

Total RNA from cultured NCI-H292 cells and animal tissues was prepared using TRIzol reagent (Invitrogen, Carlsbad, CA) according to the manufacturer's specification. The quality and quantity of RNA were determined spectrophotometrically.

\section{Reverse Transcription}

Reverse transcriptions were carried out using Super Script ${ }^{\mathrm{TM}}$ III Reverse Transcriptase (Invitrogen, Carlsbad, CA) following the manufacturer's instructions. Briefly, firststrand cDNA was synthesized in a total volume of $20 \mu \mathrm{l}$. Reactions contained total RNA $(2 \mu \mathrm{g})$, random hexamer primers $(0.25 \mu \mathrm{g})$, dNTPs $(0.5 \mathrm{mM})$, rRNasin (200 units), SuperScript ${ }^{\mathrm{TM}}$ III Reverse Transcriptase (200 units) and $5 \mathrm{x}$ first strand buffer $(4 \mu 1)$. The reactions were incubated at $65^{\circ} \mathrm{C}$ for $5 \mathrm{mins}$, followed by $1 \mathrm{~min}$ incubation on ice, 10 $\min$ at $25^{\circ} \mathrm{C}$, and 50 mins at $50^{\circ} \mathrm{C}$. Reactions were then inactivated at $70^{\circ} \mathrm{C}$ for $15 \mathrm{mins}$. 


\section{Real-Time PCR}

Quantitative real-time PCR was performed using Applied Biosystems 7500 Real Time PCR System (Foster City, CA) following the TaqMan Gene Expression Assay protocol (Applied Biosystems). TaqMan primers and probes for the human genes MUC7, MUC5AC and GAPDH (internal control), and the mice genes Muc5ac, Muc10 and Gapdh (internal control) were Pre-Developed (cataloged) by Applied Biosystems. The use of the human GAPDH and mouse Gapdh as an internal control was based on our previous findings that showed these to be optimal in our system over actin or tubulin internal controls. Each $50-\mu \mathrm{L}$ reaction (in 96-well set-up) contained the following components: 20x TaqMan Gene Expression Assay mix (2.5 $\mu \mathrm{L})$, cDNA template $+\mathrm{H}_{2} \mathrm{O}(22.5 \mu \mathrm{L}), 2 \mathrm{x}$ TaqMan Universal PCR Master Mix $(25 \mu \mathrm{L})$. The TaqMan Gene Expression Assay mix contains FAM ${ }^{\mathrm{TM}}$ dye-labeled TaqMan probe and 2 unlabeled PCR primers (the primers were designed to span intron/exon boundaries to verify that PCR products were amplified from the cDNA). To optimize the reaction, we amplified serially diluted cDNA templates that were reverse transcribed from total RNA, and plotted the log of the abundance of target gene products against $\mathrm{Ct}$ (threshold cycle). PCR amplification was carried out under the control of SDS software (Sequence Detection Systems). The data was analyzed with Relative Quantification Study Document. The relative quantity of MUC7 or MUC5AC mRNA was normalized to human GAPDH, and Muc5ac or Muc10 mRNA to mice Gapdh expression. The relative quantifications were calculated using $\triangle \triangle \mathrm{CT}$ method.

\section{Cell Lysate Preparation and ELISA Assays}

NCI-H292 cell lysates were prepared by dissolving the cells in radioimmunoprecipitation assay lysis buffer (Millipore Co, Billerica, MA). ELISA was performed with a Protein Detector ELISA kit (Kirkegaad and Perry Laboratory Inc, Gaithersburg, MD) following the manufacturer's instructions. The primary MUC7 antibody (rabbit antisera) was prepared by Bio-Synthesis (Lewisville, TX); it is specific to a 15-mer peptide derived from MUC7 mucin glycoprotein (aa 39-53). The primary MUC5AC/Muc5ac antibody was purchased from AnaSpec, Inc. (San Jose, CA). Its reactivity includes human, mouse and rat. The secondary antibody was goat anti-rabbit IgG-alkaline phosphatase conjugate. The lysates $(60 \mu \mathrm{l})$ plus coating buffer $(40 \mu 1$, included in the kit) were pipetted into 96-well microtiter plates. Human saliva $(60 \mu \mathrm{l})$ plus coating buffer $(40 \mu \mathrm{l})$ was used as a positive control, and $100 \mu \mathrm{l}$ coating buffer was used as a negative control. The plate was read at $630 \mathrm{~nm}$ using ELISA Microplate reader with 630 filter.

\section{Immunohistochemistry}

Prior to immunohistochemistry, each histological sample was incubated in $0.5 \mathrm{M}$ sucrose in PBS overnight and cryosectioned $(7 \mu \mathrm{m})$. Immediately after cryosectioning, tissue sections were washed three times in PBS (10 min at RT) and incubated with a blocking agent (1\% BSA, 10\% goat serum, $0.1 \%$ Triton X-100 in PBS) for $2 \mathrm{hrs}$ at RT. Then, they were incubated for $1 \mathrm{hr}$ at RT with monoclonal mouse anti-MUC7 antibody [14] and MUC5AC/Muc5ac monoclonal antibody (AnaSpec, Inc., Catalog\# 53684; species reactivity includes human, mouse and rat). Both antibodies were diluted 1:1000 in the blocking agent. After washing three times with PBS (10 min RT), tissue sections were incubated for $2.5 \mathrm{hrs}$ at RT with a fluorescent secondary goat anti-mouse Ig-G (Alexa Fluor 488, Molecular Probes, USA) diluted in 1\% BSA, 10\% goat serum in PBS (1:150). The sections were then washed three times in PBS (10 min each at RT), air dried for $10 \mathrm{~min}$ at RT and covered with a cover glass using an aqueous mounting medium (Immu-Mount, Thermo-Shandon, USA). Fluorescent pictures $(200 \times)$ were acquired by a digital camera (70 ms exposure time; SPOT RT-KE Diagnostic instruments Inc.), attached to a Nikon inverted fluorescent microscope (Nikon Eclipse TE2000-U).

\section{Statistical Analysis}

Statistical analyses of real-time PCR and ELISA results were performed upon comparisons made between the treated group and the control group using the Student's $t$-test. A $p$ value of less than 0.05 was considered statistically significant. Data is expressed as mean \pm standard error (SE).

\section{RESULTS}

\section{In Vitro Studies}

\section{Effects of LPS, CSE and CSE/LPS on MUC7 (and $M U C 5 A C)$ Gene Transcription}

NCI-H292 cells were selected for these experiments since it was reported in the literature that these cells express LPS receptors $[15,16]$. Quantitative real time RT-PCR was used to assess the steady-state MUC7 gene transcription. Expression of human MUC5AC, the high molecular weight respiratory mucin, shown previously to be upregulated by CSE, was also assessed for comparative purposes [7]. The results are shown in Fig. (1).

For MUC7 (Fig. 1A), a statistically significant level of induction ( $p$ value less than 0.05 ) was found with the $1 \%$ CSE treatment (3.2-fold, left panel) and with the $0.5 \% \mathrm{CSE}$ plus LPS combination treatment (4.4-fold, right panel). The $p$ value less than 0.05 was also observed for $1 \% \mathrm{CSE}$ compared with $0.5 \%$ CSE (left panel). For MUC5AC gene (Fig. 1B), the cell treatment with LPS alone resulted in the statistically significant increase of MUC5AC transcripts (6 fold, left panel). The treatment with $\mathrm{CSE}$ alone resulted in some increase of MUC5AC transcripts with each concentration of CSE used, but none of the increases were statistically significant. On the other hand, the combination of $1 \%$ CSE with LPS showed a statistically significant increase of MUC5AC transcripts (11 fold, right panel). The treatment with $2.5 \% \mathrm{CSE}$ and LPS resulted up to 23 fold increase in MUC5AC transcripts, but due to the large SD, there is no statistically significant difference compared to the untreated cells. The $p$ value less than 0.05 was also observed for $1 \%$ CSE plus LPS group compared with $0.5 \%$ CSE plus LPS group.

\section{Effects of LPS, CSE and CSE/LPS on MUC7 (and MUC5AC) Glycoprotein Production}

The levels of glycoproteins in the control and stimulated NCI-H292 cells were assessed by ELISA assay. Upon stimulation with the agents, both the MUC7 and MUC5AC 
A (MUC7)
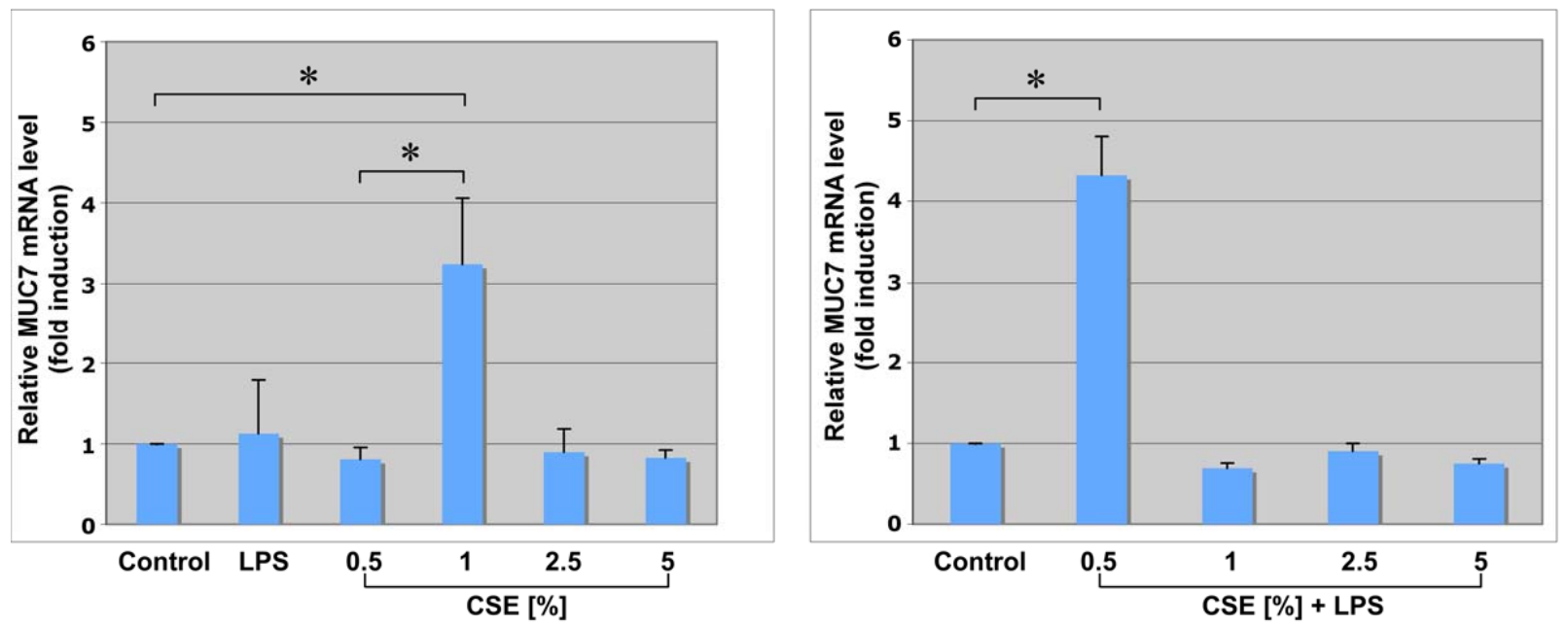

B (MUC5AC)
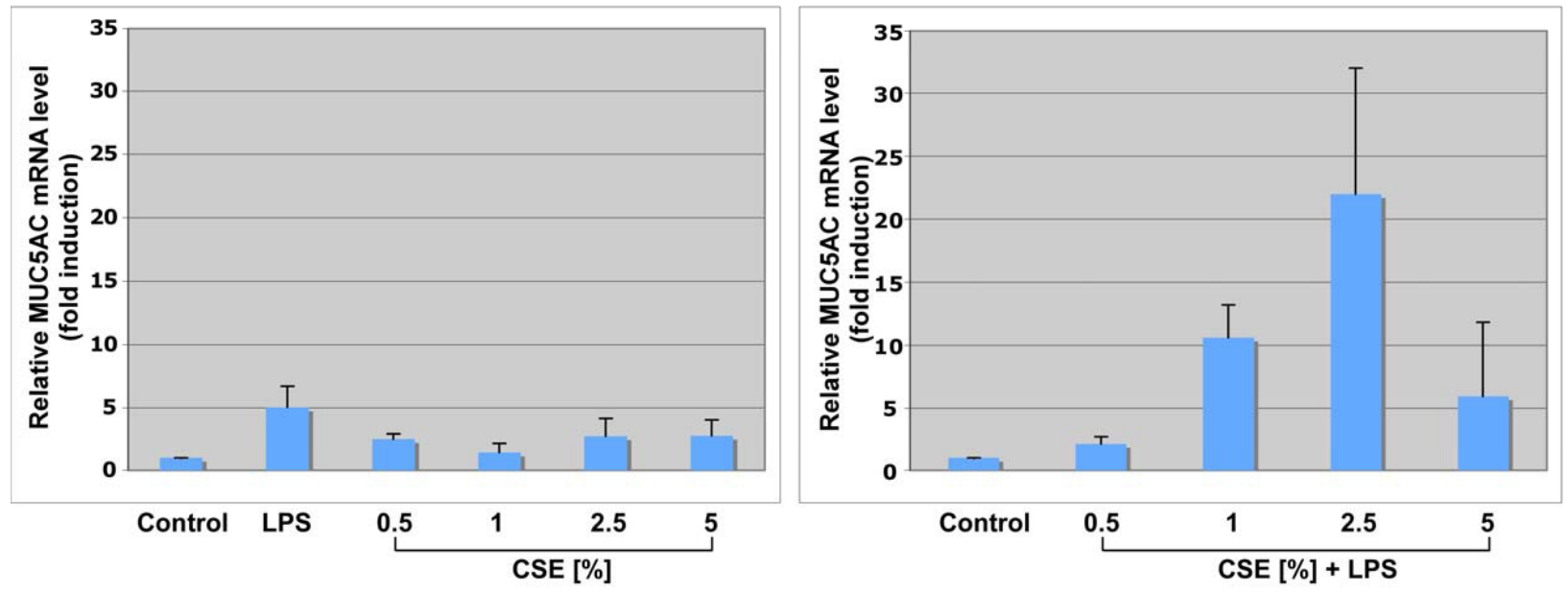

Fig. (1). Effect of CSE, LPS and CSE/LPS on mucin gene transcription in vitro, using human NCI-H292 cells. Total RNA was isolated from the control cells and treated cells and reversed transcribed. MUC7 expression (panel A) and MUC5AC expression (panel B) was determined by quantitative Real-Time PCR (qRT-PCR). The level of different mucin mRNA was normalized to GAPDH. Each bar represents the SD from three independent experiments. * indicates $p<0.05$. LPS, lipopolysaccharide from $P$. aeruginosa, used at concentration of $10 \mu \mathrm{g} / \mathrm{ml}$ in all experiments (an optimal dose, determined by the dose-response curve; data not shown). CSE, cigarette smoke extract, used at several different concentrations, either alone or in combination with LPS, as indicated. Relative levels of mucin mRNA are expressed as fold of induction comparing to the control, untreated cells, set arbitrary at a value of 1 .

mucin glycoprotein levels increased only minimally: none of the increases were statistically significant (results not shown). The MUC7 increased the most with the $0.5 \%$ CSE/LPS combination treatment (1.3 fold), and MUC5AC with the $1 \%$ CSE/LPS stimulation (1.5 fold). The other treatments resulted in smaller increase or no increase in these mucin glycoproteins.

\section{In Vivo Studies}

\section{Effect of LPS and CS on MUC7 (and Muc5ac and Muc10) Gene Transcription in MUC7 Transgenic Mice Tissues}

For these studies, we utilized the previously generated MUC7 gene transgenic mice [17]. These mice carry the human MUC7 gene $(\sim 10 \mathrm{~kb})$ with a $3 \mathrm{~kb}$ of the $5^{\prime}-$ and a 3 $\mathrm{kb}$ of the 3'-flanking sequences, totaling to a $16 \mathrm{~kb}$ of human genomic DNA. MUC7 transgene transcription in mice was assessed along with the endogenous mouse mucins, Muc5ac (high molecular weight tracheobronchial mucin) and Muc10 (ortholog of human MUC7). The results are shown in Fig. (2). In the mice trachea (Fig. 2A), the statistically significant increase $(p<0.05)$ of MUC7 transcription was observed only in the group stimulated with the LPS alone ( 2 fold); and of Muc5ac transcription in the groups stimulated with the LPS (2.5 fold), CS (2.5 fold), and CS/LPS (2.7 fold). In the mice lungs (Fig. 2B), the statistically significant increase was observed only with the MUC7 gene and only in the group of mice treated with LPS (2.1 fold). 


\section{A (trachea)}

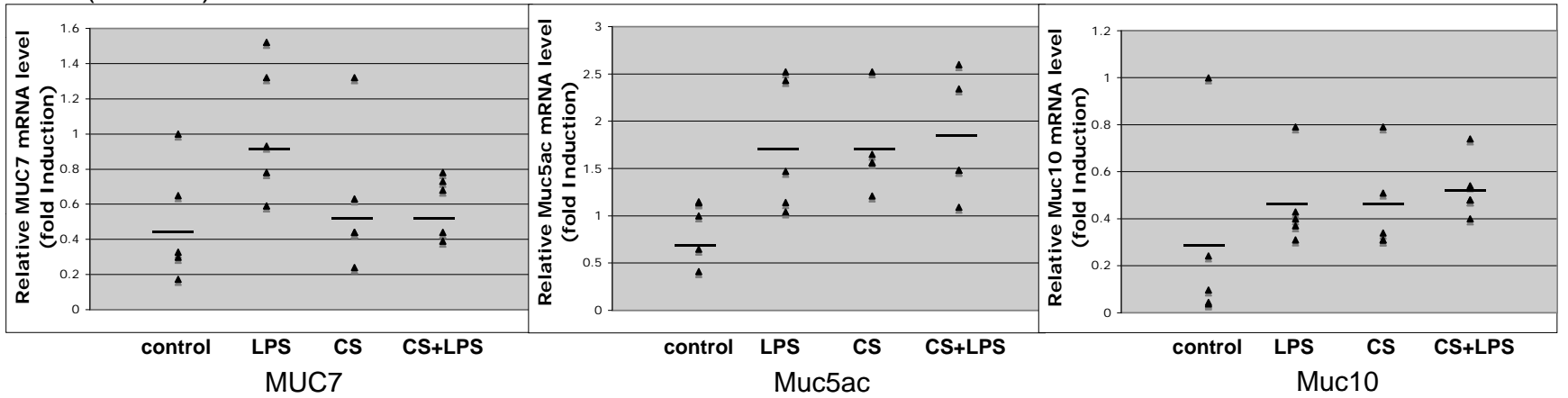

B (lungs)
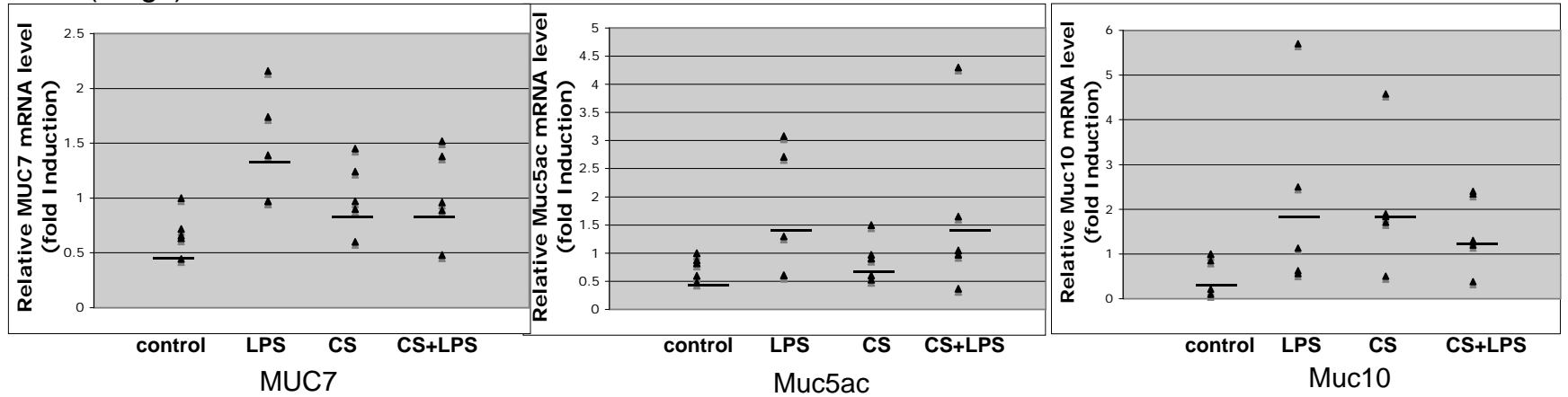

Fig. (2). Effect of CS, LPS and CS/LPS on mucin gene transcription in vivo, using MUC7 transgenic mice. Total RNA was isolated from the untreated and treated mice tissue and reverse transcribed. Human MUC7, and mouse Muc5ac and Muc10 expression in trachea (panel A) and in lung (panel B) was determined by quantitative Real-Time PCR (qRT-PCR). The level of different mucin mRNA was normalized to mouse Gapdh. Each triangle point represents individual control or experimental mouse $(\mathrm{n}=5)$. The horizontal line represents the average expression of the particular mucin in each group of animals. The mice groups are: control (exposed to air), LPS (exposed to lipopolysaccharide), CS (exposed to cigarette smoke) and CS/LPS (exposed to combination of cigarette smoke and lipopolysaccharide). The significant increase in the levels of mucin gene expression in the tissues of treated versus untreated mice, or the $p$ value $<0.05$ was found in trachea (panel A), for MUC7 with LPS treatment, and for Muc5ac with LPS, CS and CS+LPS; in lung (panel B), only for MUC7 with LPS treatment.

\section{Effect of CS and LPS on MUC7 (and Muc5ac) Glycoprotein Production in MUC7 Transgenic Mice Tissues}

In this regard, we performed immunohistochemistry (IHC) experiments with the mice trachea and lung tissue sections. In our previous study [13] we have determined by IHC which types of cells in trachea and lungs of MUC7 transgenic mice produce MUC7 glycoprotein. In trachea, MUC7 was localized within the serous cells of the submucosal glands, and in the lungs within the respiratory epithelium at the level of the bronchioles and within the stromal tissue. This study showed similar findings. The tissues of several mice were analyzed and representative results are shown in Fig. (3). The IHC data were not statistically analyzed and therefore are not quantitative. For MUC7 immunohistochemistry, a nontransgenic mice tissue was used as a negative control and the non-treated MUC7 transgenic mice tissue as a positive control. A visual examination of immunohistochemistry results from the untreated and treated animals showed the following: As expected, no detectable MUC7 glycoprotein was found in the non-transgenic mice tissue (Fig. 3A, B, Control(-)), and a positive MUC7 staining was found in the control, non-treated MUC7 transgenic mice tissue (Fig, 3A, B, Control(+)). Also, as expected, endogenous mouse Muc5ac was detected in the control, non-treated MUC7 transgenic mice tissue (Fig. 3A, B, Control panels for Muc5ac).
For the treated animals: In trachea (Fig. 3A), MUC7 glycoprotein reactivity/production appeared to increase upon mice exposure to LPS alone or to CS alone, but the highest increase was seen upon exposure to the combination of CS/LPS; Muc5ac glycoprotein reactivity/production appeared to increase upon mice exposure to CS alone and a higher increase was again seen with a combination of CS/LPS. In the lungs (Fig. 3B), MUC7 glycoprotein reactivity seemed to increase upon stimulation with the CS alone, but a much greater increase was observed with the CS/LPS combination; Muc5ac increased reactivity in lungs is observed in the mouse stimulated with CS alone or LPS alone, and the reactivity increased slightly with the CS/LPS combination exposure. Collectively, these results indicated that upon exposure of mice to a combination of CS/LPS, a MUC7 glycoprotein reactivity/production seemed to increase considerably in both trachea and lung tissues, but Muc5ac increased mostly in trachea.

\section{DISCUSSION}

\section{In Vitro Studies}

The treatment of the NCI-H292 cells with P. aeruginosa LPS did not lead to increase of MUC7 transcription despite the fact that, as reported, these cells express TLR4 $[15,16]$ through which the bacterial LPS activate cells. In our 

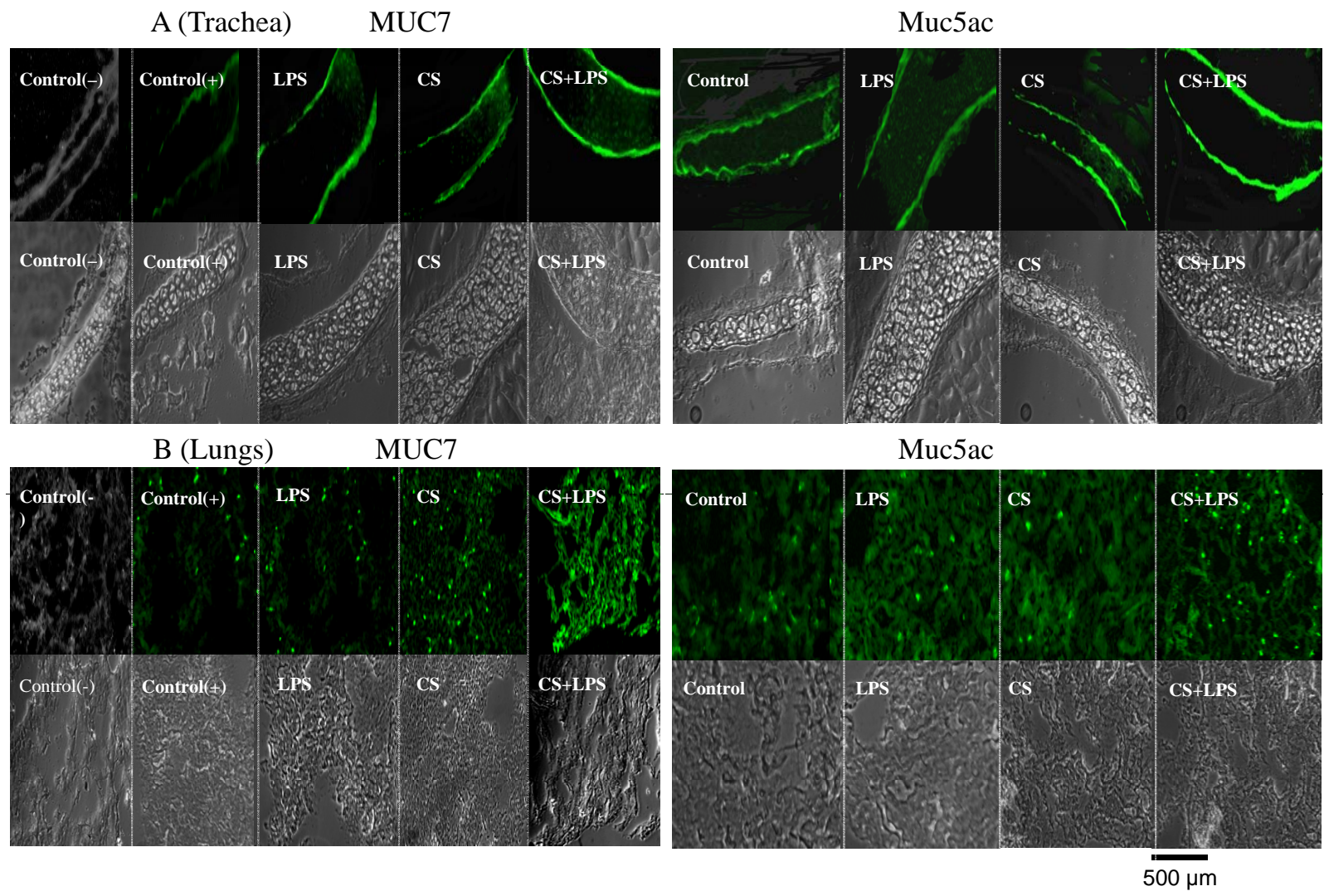

Fig. (3). Effect of CS, LPS and CS/LPS on mucin production in vivo, using MUC7 transgenic mice determined by immunohistochemistry. A representative results from tissues of several mice are shown. MUC7 and Muc5ac in trachea (panel A), and MUC7 and Muc5ac in lungs (panel B). Fluorescent (upper) and phase contrast (lower) pictures are shown for each tissue sample. For MUC7 mucin, Control(-) and Control(+) were tissue samples from trachea or lung samples from the non-treated nontransgenic mice (not expressing human MUC7) and MUC7 transgenic mice (expressing MUC7), respectively. For Muc5ac mucin control, samples from trachea or lung from the non-treated MUC7 transgenic mice (these express Muc5ac) were used. Pictures $(200 \times)$ were acquired by a digital camera attached to a Nikon inverted fluorescent microscope. Hematoxylin-eosin staining (not shown) indicated the integrity of the tissue.

previous study, we showed that MUC7 transcription was also not increased by LPS treatment of the A549 lung carcinoma cell line, but it was increased in the primary normal human tracheobronchial epithelial (NHTBE) cells [13]. We attributed the lack of MUC7 induction by the LPS in the A549 cells to the lack of the LPS receptors, TLR4 (as determined by flow cytometric analysis by another group) [18], and the MUC7 induction in the primary cells to the presence of TLR4. However, it should be noted that the more recent studies using RT-PCR showed that A549 cells do express TLR4 [16, 19]. Thus it appears that these human airway epithelial cell lines, although extensively used for in vitro studies, have a weakness, i.e. expressing variable levels of TLR4 receptor. MUC5AC transcription in the NCI-H292 cells, used in this study, increased significantly upon the LPS treatment (6-fold), and this finding is consistent with the previous studies using the same cell line [7, 20].

The treatment of NCI-H292 cells with different doses of CSE resulted in a statistically significant increase in the level of MUC7 transcription only with the $1 \%$ dose (3.2 fold). For MUC5AC, a moderate increase was seen with the most CSE doses used, but none of the increases were statistically significant. The previous studies indicated that MUC5AC was upregulated by CSE in NCI-H292 cells [7] and further that cigarette smoke causes mucin overproduction via EGF receptor (EGFR) activation in airway epithelial cells in vitro and in vivo $[21,22]$.

Regarding the question whether the MUC7 gene transcription can be enhanced by the LPS/CSE combination treatment in NCI-H292 cell line, we found that MUC7 mRNA was statistically significantly increased only with the $0.5 \% \mathrm{CSE} / \mathrm{LPS}$ treatment. In fact, the MUC7 transcript was increased by 4.4 fold, which represents a synergistic increase. However, $1 \%$ CSE/LPS treatment led to no MUC7 transcription increase, and in fact the level was even lower than for the untreated, control cells, indicating that this combination lead to antagonistic effect, eliminating the 3.2fold increase achieved with $1 \%$ CSE. A suppression of MUC7 expression may not be due to potential toxicity of the combined stimuli, because stimulation of cells with $1 \%$ CSE/LPS treatment yielded 11-fold transcription increase of MUC5AC. This latter finding also represents a synergistic effect and it is in an agreement with the previous study, which demonstrated that co-stimulation with $1 \%$ CSE/LPS 
synergistically increased MUC5AC mucin transcription (15fold) in NCI-H292 cells [7]. The underlying mechanisms of synergy between the CSE and LPS are unclear, but the authors of the above study speculated that CSE acts at multiple levels to synergistically enhance mucin production. We have noticed a recent study that reported that CSE induced expression of TLR4 and LPS binding in another epithelial cell line, namely 16-HBE [23], and thus it is tempting to speculate that this could be one possible explanation for the observed synergy between the CSE and LPS treatment of NCI-H292 cells and the transcription of MUC7 and MUC5AC.

The CS, LPS, and the combination of CS/LPS treatment of the NCI-H292 cell line resulted only in a small increase in both MUC7 glycoprotein (maximum 1.3 fold with the $0.5 \%$ CSE/LPS treatment) and MUC5AC glycoprotein production (maximum 1.5 fold with the 1\% CSE/LPS treatment; data not shown). Note, however, that both of these highest increases in glycoprotein production were achieved with the same combination of CSE/LPS treatment that yielded the synergistic increase of the MUC7 and MUC5AC transcripts, indicating some degree of correlation between transcription and glycoprotein production. Other studies, using the same cell line, reported elevation of MUC5AC glycoprotein upon CSE treatment, which appeared to increase in a dose dependent manner, from 1.3-2.5 fold [24] and from 2-3 fold [7], and with the combination of CSE/LPS treatment, 4-fold induction was seen [7].

\section{In Vivo Studies}

We chose to use CS rather than CSE for in vivo experiments in order to resemble the smoke exposure experience by smokers. Even though intranasal administration of CSE in vivo maybe more convenient, the study by Elliot et al. [25] showed that its resemblance to the CS exposure experience is not equivalent. CSE resulted in a heavier exposure to cigarette smoke components over the same treatment length.

Measuring induction of human MUC7 mRNA after treatment of MUC7 transgenic mice with LPS, CS or combination of LPS/CS showed statistically significant increase in MUC7 mRNA in both trachea and lung with LPS treatment only (Fig. 2). The two-fold increase of MUC7 mRNA with the LPS treatment is consistent with our previous in vivo study [13]. Mouse Muc5ac transcript was found to statistically increase in trachea (but not in lungs) with all three treatments (LPS, CS, LPS/CS), compared to the control animals. On the other hand, the study [7] using a rat model showed no induction of rat muc5AC with LPS or CS alone, in either trachea or lungs; the induction was seen only with the combination of LPS/CS, in both trachea and lungs.

The levels of MUC7 or Muc5a glycoprotein reactivity/ production in the animal tissues using immunohistochemistry in our study, and the level of total mucin content in the tracheobronchial lavage fluid measured by enzymelinked lectin assay by the investigators using the rat model [7], are hard to compare. Also, our immunohistochemistry results are not quantitative. Nevertheless, the exposure of animals to a combination of LPS/CS treatment seems to result in increased levels of MUC7 and Muc5ac mucin reactivity/production in our mouse model, and increased the total mucin levels in the rat model, as compared to the animals treated with CS or LPS alone.

The role of MUC7 and its regulation in different stages of different diseases is still not understood. MUC7 is a small, soluble, non-gel-forming mucin. Its main function is thought to be clearance of various bacteria and thus inhibition of bacterial colonization. It has been shown to interact in vitro with many oral and respiratory microorganisms, including $P$. aeruginosa [26]. Thus, the increased production of MUC7 soluble mucin, seen upon exposure of mice to $P$. aeruginosa LPS/CS exposure shown in vivo by immunohistochemistry in this study, may reduce the viscosity of the mucus caused by overproduction of the large, gel forming mucins in the respiratory track. This increase in the soluble MUC7 may facilitate the mucocilliary clearance, and thus have a protective role in the respiratory track by maintaining an appropriate balance between detrimental and beneficial outcomes.

\section{CONCLUSIONS}

$1 \% \mathrm{CSE}$ and $0.5 \% \mathrm{CSE} / \mathrm{LPS}$ treatment of the cell line resulted in statistically significant and synergistic increase of MUC7 transcript, respectively. The exposure of mice to CS, LPS or CS/LPS resulted in statistically significant increase in MUC7 transcripts in both trachea and lung with LPS only, and a variable increase of MUC7 glycoprotein, with the highest increase with CS/LPS. These results contributed to the understanding of the regulation of MUC7 expression by pathogenic factors, however, further studies are necessary in order to understand the mechanism of MUC7 regulation and its possible role in different stages of different diseases. The possible protective role of the increased soluble MUC7 in the respiratory tract induced by CS/LPS warrants further investigation.

\section{ACKNOWLEDGEMENTS}

We thank Dr. G. Intini and J. Amarasigne for valuable advice on immunohistochemistry and qRT-PCR, respectively. We also acknowledge Dr. H. Yao for help with the control qRT-PCR, and Dr. M.I. Cho for help with interpretation of immunohistochemistry slides.

\section{DECLARATION OF INTERESTS}

The authors report no declarations of interest. This study was supported by the NIH/NIDCR RO1 grand DE009820 (LAB).

\section{ABBREVIATIONS \\ LPS = Pseudomonas aeruginosa lipopolysaccharide \\ $\mathrm{CSE}=$ Cigarette smoke extract \\ $\mathrm{CS}=$ Cigarette smoke}

\section{REFERENCES}

[1] Ali MS, Pearson JP. Upper airway mucin gene expression: a review. Laryngoscope 2007; 117: 932-8.

[2] Rose MC, Voynow JA. Respiratory tract mucin genes and mucin glycoproteins in health and disease. Physiol Rev 2006; 86: 245-78.

[3] McNamara N, Basbaum C. Signaling networks controlling mucin production in response to Gram-positive and Gram-negative bacteria. Glycoconj J 2001; 18: 715-22. 
[4] Macnee W, Rahman I. Oxidants and antioxidants as therapeutic targets in chronic obstructive pulmonary disease. Am J Respir Crit Care Med 1999; 160: S58-65.

[5] Jiao ZX, Ao QL, Xiong M. Cigarette smoke extract inhibits the proliferation of alveolar epithelial cells and induces apoptosis. Sheng Li Xue Bao 2006; 58: 244-54.

[6] Marwick JA, Stevenson CS, Giddings J, et al. Cigarette smoke disrupts VEGF165-VEGFR-2 receptor signaling complex in rat lungs and patients with COPD: morphological impact of VEGFR-2 inhibition. Am J Physiol Lung Cell Mol Physiol 2006; 290: L897908.

[7] Baginski TK, Dabbagh K, Satjawatcharaphong C, Swinney DC. Cigarette smoke synergistically enhances respiratory mucin induction by proinflammatory stimuli. Am J Respir Cell Mol Biol 2006; 35: 165-74.

[8] Biesbrock AR, Bobek LA, Levine MJ. MUC7 gene expression and genetic polymorphism. Glycoconj J 1997; 14: 415-22.

[9] Bobek LA, Tsai H, Biesbrock AR, Levine MJ. Molecular cloning, sequence, and specificity of expression of the gene encoding the low molecular weight human salivary mucin (MUC7). J Biol Chem 1993; 268: 20563-9.

[10] Sharma P, Dudus L, Nielsen PA, et al. MUC5B and MUC7 are differentially expressed in mucous and serous cells of submucosal glands in human bronchial airways. Am J Respir Cell Mol Biol 1998; 19: 30-7.

[11] Schenkels LCPM, Gururaja TL, Levine MJ. In: Rathbone MJ, Ed. Salivary mucins: their role in oral mucosal barrier function and drug delivery. Hamilton, New Zeland: Marcel Dekker, Inc; 1996; 191-220.

[12] Tabak LA. In defense of the oral cavity: structure, biosynthesis, and function of salivary mucins. Annu Rev Physiol 1995; 57: 54764.

[13] Li S, Intini G, Bobek LA. Modulation of MUC7 mucin expression by exogenous factors in airway cells in vitro and in vivo. Am J Respir Cell Mol Biol 2006; 35: 95-102.

[14] Cohen RE, Aguirre A, Neiders ME, et al. Immunochemistry and immunogenicity of low molecular weight human salivary mucin. Arch Oral Biol 1991; 36: 347-56
[15] Gon Y, Asai Y, Hashimoto S, et al. A20 inhibits toll-like receptor 2- and 4-mediated interleukin-8 synthesis in airway epithelial cells. Am J Respir Cell Mol Biol 2004; 31: 330-6.

[16] Hou YF, Zhou YC, Zheng XX, et al. Modulation of expression and function of Toll-like receptor 3 in A549 and H292 cells by histamine. Mol Immunol 2006; 43: 1982-92.

[17] Bobek LA, Li H, Rojstaczer N, Jones C, Gross KW, Levine MJ. Tissue-specific expression of human salivary mucin gene, MUC7, in transgenic mice. Transgenic Res 1998; 7: 195-204.

[18] Tsutsumi-Ishii Y, Nagaoka I. Modulation of human beta-defensin-2 transcription in pulmonary epithelial cells by lipopolysaccharidestimulated mononuclear phagocytes via proinflammatory cytokine production. J Immunol 2003; 170: 4226-36.

[19] Zhou M, Wan HY, Huang SG, Li B, Li M. [Expression of toll-like receptor 4 in human alveolar epithelial cells and its role in cellular inflammation]. Zhonghua Yi Xue Za Zhi 2008; 88: 2112-6.

[20] Kohri K, Ueki IF, Shim JJ, et al. Pseudomonas aeruginosa induces MUC5AC production via epidermal growth factor receptor. Eur Respir J 2002; 20: 1263-70.

[21] Takeyama K, Dabbagh K, Lee HM, et al. Epidermal growth factor system regulates mucin production in airways. Proc Natl Acad Sci USA 1999; 96: 3081-6.

[22] Takeyama K, Jung B, Shim JJ, et al. Activation of epidermal growth factor receptors is responsible for mucin synthesis induced by cigarette smoke. Am J Physiol Lung Cell Mol Physiol 2001; 280: L165-72.

[23] Pace E, Ferraro M, Siena L, et al. Cigarette smoke increases Tolllike receptor 4 and modifies lipopolysaccharide-mediated responses in airway epithelial cells. Immunology 2008; 124: 401-11.

[24] Lee SY, Kang EJ, Hur GY, et al. The inhibitory effects of rebamipide on cigarette smoke-induced airway mucin production. Respir Med 2006; 100: 503-11.

[25] Elliott MK, Sisson JH, West WW, Wyatt TA. Differential in vivo effects of whole cigarette smoke exposure versus cigarette smoke extract on mouse ciliated tracheal epithelium. Exp Lung Res 2006; 32: 99-118.

[26] Tabak LA. In defense of the oral cavity: the protective role of the salivary secretions. Pediatr Dent 2006; 28: 110-7; discussion 92-8.

(C) Fan and Bobek; Licensee Bentham Open.

This is an open access article licensed under the terms of the Creative Commons Attribution Non-Commercial License (http://creativecommons.org/licenses/ by-nc/3.0/) which permits unrestricted, non-commercial use, distribution and reproduction in any medium, provided the work is properly cited. 